\title{
Weak Limits of Zeros of Orthogonal Polynomials
}

\author{
J. L. Ullman and M. F. Wyneken
}

\begin{abstract}
Let $\mu$ be a positive unit Borel measure with infinite support on the interval $[-1,1]$. Let $P_{n}(x, \mu)$ denote the monic orthogonal polynomial of degree $n$ associated with $\mu$, and let $\nu_{n}(\mu)$ denote the unit measure with mass $1 / n$ at each zero of $P_{n}(x, \mu)$. A carrier is a Borel subset of the support of $\mu$ having unit $\mu$-measure, and a measure $\nu$ is carrier related to $\mu$ when it has the same carriers as $\mu$. We demonstrate that for each carrier $B$ of positive capacity there is a measure $\nu$, which is carrier related to $\mu$, such that the equilibrium measure of the carrier $B$ is the weak limit of the sequence $\left\{\nu_{n}(\nu)\right\}_{n=1}^{\infty}$.
\end{abstract}

\section{Introduction}

Let $\mu$ be a positive unit Borel measure with infinite support on the interval $I=[-1,1]$. Let $P_{n}(x, \mu)$ denote the monic orthogonal polynomial of degree $n$ associated with $\mu$, so that

$$
\left(\int P_{m}(x, \mu) P_{n}(x, \mu) d \mu\right)^{1 / 2}=N_{n}(\mu) \delta_{m n}, \quad \delta_{m n}=\left\{\begin{array}{ll}
0 & \text { if } m \neq n \\
1 & \text { if } m=n
\end{array},\right.
$$

and $N_{n}(\mu)$ is the $L^{2}$-norm. The polynomial $P_{n}(x, \mu)$ has $n$ simple zeros on $I$. Let $\nu_{n}=\nu_{n}(\mu)$ denote the zero measure of $P_{n}(x, \mu)$, i.e., the unit measure with mass $1 / n$ at each zero of $P_{n}(x, \mu)$. If for a unit Borel measure $\nu$ defined on $I$ we have $\lim _{n \rightarrow \infty} \int f d \nu_{n}=\int f d \nu$ for all functions $f(x)$ continuous on $I$, we say that the sequence $\left\{\nu_{n}\right\}_{n=1}^{\infty}$ converges weakly to $\nu$, or $\nu$ is a weak limit of $\left\{\nu_{n}\right\}_{n=1}^{\infty}$, and we write $\lim _{n \rightarrow \infty} \nu_{n}=\nu$. By a theorem of Helly, $\left\{\nu_{n}\right\}_{n=1}^{\infty}$ always has weakly convergent subsequences.

In this paper we demonstrate that the equilibrium measures of the carriers are weak limits of the zero measures of the orthogonal polynomials associated with the so-called carrier related measures. A carrier is a Borel subset of the support having unit measure, and two measures are carrier related when they share the same set of carriers. An equilibrium measure is a potential theoretic measure which in the instance of a compact set is energy minimizing.

Other related results and open questions will be discussed in Section 5.

Date received: January 22, 1986. Date revised: March 19, 1986. Communicated by Edward B. Saff. AMS classification: 30C15, 31C15, 33A65, 42C05.

Key words and phrases: Borel measure, Weak limits of orthogonal polynomials, Carrier, Equilibrium measure. 


\section{Statement of Theorem}

The support $S(\mu)$ of a positive unit Borel measure $\mu$ on $I$ is the smallest closed set of unit $\mu$-measure. When $S(\mu)$ is an infinite set such a measure $\mu$ is called a weight measure.

The capacity of a Borel set $B$ is taken as its inner capacity

$$
C(B)=\sup _{K \in B} C(K),
$$

where $K$ is a compact subset of $B$, and $C(K)$ is the capacity of $K$ as derived from the logarithmic potential function [5]

$$
U(z, \mu)=\int \log \left(|z-t|^{-1}\right) d \mu(t) .
$$

The following are properties of capacity [5]: for Borel sets $B_{1}$ and $B_{2}$,

(i) $B_{1} \subset B_{2} \Rightarrow C\left(B_{1}\right) \leq C\left(B_{2}\right)$,

(ii) $C\left(B_{1}\right)=0 \Rightarrow C\left(B_{2} \cup B_{1}\right)=C\left(B_{2}-B_{1}\right)=C\left(B_{2}\right)$,

(iii) for compact $K_{n} \subset K_{n+1} \subset I(n=1,2, \ldots), \lim _{n \rightarrow \infty} C\left(K_{n}\right)=C\left(\bigcup_{n=1}^{\infty} K_{n}\right)$.

Associated with a set of carrier related measures are two numbers,

$$
\underline{C}=\inf C(B), \quad \bar{C}=\sup C(B),
$$

taken over the carriers $B$. When $C=\bar{C}$ we say that $\mu$ is a determined measure, and undetermined otherwise. To see the existence of an undetermined measure let $O$ be a dense open subset of $I$ with $C(O)=\lambda<\frac{1}{2}$. Let $f(x)$ be a positive, Lebesgue integrable function defined on $O$ with $\int_{o} f d x=1$. If we define a measure $\mu$ by $\mu(B)=\int_{B \cap o} f d x$ for Borel sets $B$, then $\mu$ is a unit measure with $C=\lambda$ and $\bar{C}=\frac{1}{2}[5]$.

The equilibrium measure for a bounded Borel set $B$ of positive capacity is the unique measure $\mu_{B}$ which satisfies the following [3, p. 92]:

(i) $S\left(\mu_{B}\right)$ is bounded,

(ii) $U\left(z, \mu_{B}\right) \leq \log \left(\left(C(B)^{-1}\right)\right.$ for all $z \in \mathbf{C}$.

(iii) $U\left(z, \mu_{B}\right)=\log \left(\left(C(B)^{-1}\right)\right.$ for all $z$ in a Borel subset $B^{*}$ of $B$ of the same capacity.

We now state the theorem which we will prove in the next section.

Theorem. Let $\mu$ be an undetermined weight measure with $\underline{C}>0$, and let $B$ be any carrier of $\mu$. Then there exists a carrier related measure $\nu$ such that

$$
\lim _{n \rightarrow \infty} \nu_{n}(\nu)=\mu_{B}
$$

and

$$
\lim _{n \rightarrow \infty}\left(N_{n}(\nu)\right)^{1 / n}=C(B) .
$$


We conclude this section by stating three other results on weak limits due to the first author whose proofs will appear elsewhere. Let $\mu$ be a weight measure with $\underline{C}>0$. Let $\bar{\mu}$ denote the equilibrium measure of the support, let $\mu$ denote the equilibrium measure which is common to the carriers whose capacity is $\underline{C}$, and let $\lambda_{n}(\nu)=\left(\int\left(P_{n}(x, \nu)\right)^{2} d \nu\right)^{1 / 2 n}$.

A. If $\mu$ is determined, then for any carrier related measure $\nu$,

$$
\lim _{n \rightarrow \infty} \nu_{n}(\nu)=\bar{\mu} .
$$

B. If $\mu$ is undetermined, then for a carrier related measure $\nu$,

$$
\begin{aligned}
& \lim _{n \rightarrow \infty} \lambda_{n}(\nu)=\underline{C} \Rightarrow \lim _{n \rightarrow \infty} \nu_{n}(\nu)=\underline{\mu}, \\
& \lim _{n \rightarrow \infty} \lambda_{n}(\nu)=\bar{C} \Rightarrow \lim _{n \rightarrow \infty} \nu_{n}(\nu)=\bar{\mu} .
\end{aligned}
$$

C. If $\mu$ is undetermined, and $\nu$ is any weak limit of the zero measures of some carrier related measure, then

$$
\bar{\mu}(B) \leq \nu(B) \leq \underline{\mu}(B)
$$

for any Borel subset $B$ of $\left\{z: U(z, \underline{\mu})=\log \left(\underline{C}^{-1}\right)\right\}$.

\section{Proof of Theorem}

The property of being carrier related is equivalent to being mutually absolutely continuous [7], and hence it will suffice to construct a positive a.e. $\mu$ Borel measurable function $\omega(x), \int \omega(x) d \mu=1$, such that $d \nu=\omega(x) d \mu$ yields the requisite property.

We now state three lemmas. Lemmas 2 and 3 will be proved in Section 4, and Lemma 1 is due to Szegö and Tonelli.

Lemma $1([4$, p. 73$])$. Let $K$ be a compact subset of $I$, and let $M_{n}(K)=\inf \left\|P_{n}(x)\right\|_{K}$ where the infimum is taken over all monic polynomials $P_{n}(x)$ of degree $n$, and $\left\|P_{n}(x)\right\|_{K}=\max \left|P_{n}(x)\right|$ over $x \in K$.

(i) If $K$ contains at least $n$ points, then there is a unique monic Chebychev polynomial $T_{n}(x, K)$ of degree $n$ satisfying $\left\|T_{n}(x, K)\right\|_{K}=M_{n}(K)$.

(ii) If $K$ is infinite, then $\lim _{n \rightarrow \infty}\left(M_{n}(K)\right)^{1 / n}=C(K)$.

(iii) For $x \in I,\left|T_{n}(x, K)\right| \leq 2^{n}$.

Let $K$ be a compact set of positive capacity and let $\Omega$ be the unbounded component of the complement of $K$ in $\mathbf{C}$. The potential function $U\left(z, \mu_{K}\right)$ is harmonic for $z \in \Omega$, and when it is continuous on $\mathbf{C}$ we say that $K$ is regular. Ancona [1], [2] has shown that each Borel set $B$ with $C(B)>\varepsilon>0$ has a regular compact subset $\mathscr{K}$ such that $C(\mathscr{K})>C(B)-\delta$ for each value of $\delta$ where $0<\delta<\varepsilon$.

Let $N_{\varepsilon}(z)$ denote the $\varepsilon$-neighborhood of $z$, i.e., $N_{\varepsilon}(z)=\{w:|w-z|<\varepsilon\}$. 
Lemma 2 ([6]). Let $\mu$ be a weight measure, let $B$ be a Borel subset of $S(\mu)$, and let $\mathscr{K}$ be a regular compact subset of $S(\mu)$. Assume that $\mu\left(B \cap N_{\varepsilon}(x)\right)>0$ for each $x \in \mathscr{K}$ and all $\varepsilon>0$. Then

(i) for each positive integer $n$, there is a nonnegative Borel measurable function $\tau_{n}(x)$, called a transfer function, with the properties that $\int \tau_{n}(x) d \mu=n^{-2}$ and $A_{n}=\left\{x: \tau_{n}(x)>0\right\}$ is a compact subset of $B$, and

(ii) there is a sequence of positive numbers $\left\{c_{n}\right\}_{n=1}^{\infty}$ depending only on $\mathscr{K}$ with the property that $\lim _{n \rightarrow \infty}\left(c_{n}\right)^{1 / n}=1$ and such that if $P_{n}(x)$ is any polynomial of degree $n$, then

$$
\int\left(P_{n}(x)\right)^{2} \tau_{n}(x) d \mu \geq c_{n}\left\|P_{n}(x)\right\|_{\mathscr{K}}^{2} .
$$

Lemma 3. Let $\mu$ be a weight measure, let $\nu$ be a carrier related measure, and let $B$ be a carrier of positive capacity $\lambda$. Then the following two statements are equivalent:

$$
\begin{aligned}
\lim _{n \rightarrow \infty} \nu_{n}(\nu) & =\mu_{B}, \\
\varlimsup_{n \rightarrow \infty}\left|P_{n}(x, \nu)\right|^{1 / n} & \leq \lambda
\end{aligned}
$$

for all $x$ in some Borel subset $B^{*}$ of $B$ of the same capacity.

Now assume $C(B)=\lambda$. Let $\left\{\varepsilon_{p}\right\}_{p=0}^{\infty}$ be a sequence of positive numbers decreasing to zero. For each integer $p(p=1,2, \ldots)$ we may assume there exists a regular compact set $\mathscr{K}_{p}$ and a compact set $K_{p}$ with $\mathscr{K}_{p} \subset K_{p} \subset B, C\left(\mathscr{K}_{p}\right)>\max \left(\lambda-\varepsilon_{p}, 0\right)$ and $\mu\left(K_{p}\right)>\max \left(1-\varepsilon_{p}, 0\right)$. Moreover, we may assume $K_{p} \subset K_{p+1}$, and from the Wiener criterion $\left[4\right.$, p. 104] we may assume $\mathscr{K}_{p} \subset \mathscr{K}_{p+1}(p=1,2, \ldots)$.

Let $K_{p, \delta}=\left\{x \in \mathbf{R}\right.$ : there exists $y \in K_{p}$ such that $\left.|x-y| \leq \delta\right\}$. Let $\delta_{p} \quad(p=$ $-1,0,1,2, \ldots)$ denote positive numbers whose values will be determined recursively. Let $B_{p}=K_{p, \delta_{p-2}} \cap K_{p+1, \delta_{p-1}} \cap B(p=1,2, \ldots)$. By Lemma 2 there exists for each integer $n$ a transfer function $\tau_{p, n}(x)$ supported on a compact subset $A_{p, n}$ of $B_{p}$, such that for any polynomial $P_{n}(x)$ of degree $n$

$$
\int\left(P_{n}(x)\right)^{2} \tau_{p, n}(x) d \mu \geq c_{p, n}\left\|P_{n}(x)\right\|_{\mathscr{K}_{p}}^{2}
$$

with $\lim _{n \rightarrow \infty}\left(c_{p, n}\right)^{1 / n}=1$.

Let $c_{p}$ be an integer such that $n>c_{p}$ implies $\left(c_{p, n}\right)^{1 / n} \geq 1-\varepsilon_{p}(p=1,2, \ldots)$. For an infinite compact set $L$ with $C(L)<\lambda+\varepsilon$, let $[L, \varepsilon]$ denote the least positive integer for which $n>[L, \varepsilon]$ implies $\left\|T_{n}(x, L)\right\|_{L} \leq(\lambda+\varepsilon)^{n}$; this exists by Lemma 1. Let $[\Sigma, s]$ denote the least positive integer $m$ for which $\sum_{n>m} n^{-2} \leq(\lambda / 2)^{2 s}$.

We now describe the beginning and general steps of the recursive construction. Let $s_{0}=t_{0}=0, A_{0}=\varnothing, \delta_{-1}=\delta_{0}=1$ and $L_{0}=K_{2, \delta_{0}} \cup A_{0}$. For the first stage let $s_{1}=\max \left(\left[L_{0}, \varepsilon_{0}\right], s_{0}+1\right), t_{1}=\max \left(\left[\Sigma, s_{1}\right], c_{2}, t_{0}+1\right)$. Then choose $A_{1,1}, \ldots, A_{1, t_{1}}$ and let $A_{1}=A_{1,1} \cup \cdots \cup A_{1, t_{1}} \cup A_{0}$. Finally, define $\delta_{1}, L_{1}$, by $L_{1}=K_{3, \delta_{1}} \cup A_{1}$ so that $C\left(L_{1}\right)<\lambda+\varepsilon_{1}[3$, p. 86]

Before starting the $p$ th stage, we have determined $s_{k}, t_{k}, A_{k}, L_{k}$, and $\delta_{k}, k=$ 
$1,2, \ldots, p-1$. We then let $s_{p}=\max \left(\left[L_{p-1}, \varepsilon_{p-1}\right], s_{p-1}+1\right), t_{p}=\max \left(\left[\Sigma, s_{p}\right], c_{p+1}\right.$, $\left.t_{p-1}+1\right)$. Then choose $A_{p, t_{p-1}}+1, \ldots, A_{p, t_{p}}$ and let $A_{p}=A_{p, t_{p-1}+1} \cup \cdots \cup A_{p, t_{p}} \cup$ $A_{p-1}$. Finally, define $\delta_{p}, L_{p}$, by $L_{p}=K_{p+2, \delta_{p}} \cup A_{p}$ so that $C\left(L_{p}\right)<\lambda+\varepsilon_{p}$.

Thus by recursive definition we have established the existence of sequences $\left\{s_{k}\right\},\left\{t_{k}\right\},\left\{A_{k}\right\},\left\{L_{k}\right\}$, and $\left\{\delta_{k}\right\}(k=0,1, \ldots)$ with the properties

(a) $\left\{s_{k}\right\}$ is an increasing sequence,

(b) $s_{k} \geq\left[L_{k-1}, \varepsilon_{k-1}\right]$ for $k \geq 1$,

(c) $\left\{t_{k}\right\}$ is an increasing sequence,

(d) $t_{k} \geq c_{k+1}$ for $k \geq 1$,

(e) $t_{k} \geq\left[\Sigma, s_{k}\right]$ for $k \geq 1$,

(f) $A_{k+2} \subset L_{k}$ for $k \geq 0$, since $A_{k} \subset L_{k}, A_{k+1, n} \subset B_{k+1} \subset K_{k+2, \delta_{k}} \subset L_{k}$ for $t_{k}+1 \leq$ $n \leq t_{k+1}$, and $A_{k+2, n} \subset B_{k+2} \subset K_{k+2, \delta_{k}} \subset L_{k}$ for $t_{k+1}+1 \leq n \leq t_{k+2}$,

(g) $K_{k+2} \subset L_{k}$ for $k \geq 0$, and

(h) $C\left(L_{k}\right)<\lambda+\varepsilon_{k}$ for $k \geq 1$.

We refer to these as properties (a), (b), etc., later in this section. Then let

$$
w_{p}(x)=\chi_{K_{p}}(x)\left(\mu\left(K_{p}\right)\right)^{-1}\left((\lambda / 2)^{2 s_{p-1}}-(\lambda / 2)^{2 s_{p}}\right) \text {, }
$$

where $\chi_{K_{p}}(x)$ is the characteristic function of the set $K_{p}$, and let

$$
\omega(x)=c \sum_{p=1}^{\infty} w_{p}(x)+c \sum_{p=1}^{\infty} \sum_{t_{p-1}<n \leq t_{p}} \tau_{p, n}(x)=W_{1}(x)+W_{2}(x),
$$

where $c$ is chosen so that $\int \omega(x) d \mu=1$. Note that $W_{1}(x)>0$ a.e. $\mu$.

Then for $s_{p+1}<n \leq s_{p+2}, p=1,2, \ldots$,

$$
\begin{aligned}
N_{n}^{2}(\nu)= & \int\left(P_{n}(x, \nu)\right)^{2} d \nu \\
= & \int\left(P_{n}(x, \nu)\right)^{2} \omega(x) d \mu \\
\leq & \int\left(T_{n}\left(x, L_{p}\right)\right)^{2} \omega(x) d \mu \quad([5]) \\
= & \int_{L_{p}}\left(T_{n}\left(x, L_{p}\right)\right)^{2} \omega(x) d \mu+\int_{S(\mu)-L_{p}}\left(T_{n}\left(x, L_{p}\right)\right)^{2} W_{1}(x) d \mu \\
& +\int_{S(\mu)-L_{p}}\left(T_{n}\left(x, L_{p}\right)\right)^{2} W_{2}(x) d \mu \\
= & I_{1}+I_{2}+I_{3} .
\end{aligned}
$$

Now $I_{1} \leq\left(\lambda+\varepsilon_{p}\right)^{2 n}$ (properties (b) and (h)).

Next we have

$$
\begin{aligned}
I_{2} & \leq 2^{2 n} \int_{S(\mu)-L_{p}} W_{1}(x) d \mu \\
& \leq c 2^{2 n} \int_{\cup_{k=p+3}^{\infty}\left(K_{k}-K_{p+2}\right)} \sum_{k=1}^{\infty} \chi_{K_{k}}(x)\left(\mu\left(K_{k}\right)\right)^{-1}\left((\lambda / 2)^{2 s_{k-1}}-(\lambda / 2)^{2 s_{k}}\right) d \mu
\end{aligned}
$$




$$
\begin{aligned}
& =c 2^{2 n} \sum_{k=1}^{\infty} \mu\left(K_{k}-K_{p+2}\right)\left(\mu\left(K_{k}\right)\right)^{-1}\left((\lambda / 2)^{2 s_{k-1}}-(\lambda / 2)^{2 s_{k}}\right) \\
& \leq c 2^{2 n} \sum_{k=p+3}^{\infty}\left((\lambda / 2)^{2 s_{k-1}}-(\lambda / 2)^{2 s_{k}}\right) \\
& =c 2^{2 n}(\lambda / 2)^{2 s_{p+2}} \\
& \leq c \lambda^{2 s_{p+2}} \\
& \leq c \lambda^{2 n}
\end{aligned}
$$

Finally,

$$
\begin{aligned}
I_{3} & \leq 2^{2 n} \int_{S(\mu)-L_{p}} W_{2}(x) d \mu \\
& \leq c 2^{2 n} \int_{\bigcup_{k=1}^{\infty}\left(A_{k}-A_{k-1}-L_{p}\right)} \sum_{p=1}^{\infty} \sum_{t_{p-1}<n \leq t_{p}} \tau_{p, n}(x) d \mu \\
& \leq c 2^{2 n} \int_{\bigcup_{k=p+3}^{\infty}\left(A_{k}-A_{k-1}\right)} \sum_{p=1}^{\infty} \sum_{t_{p-1}<n \leq t_{p}} \tau_{p, n}(x) d \mu \quad \text { (property (f)) } \\
& \leq c 2^{2 n} \sum_{n=t_{p+2}+1}^{\infty} n^{-2} \\
& \leq c 2^{2 n}(\lambda / 2)^{2 s_{p+2}} \quad(\text { property }(\mathrm{e})) \\
& \leq c \lambda^{2 s_{p+2}} \\
& \leq c \lambda^{2 n} .
\end{aligned}
$$

Thus $N_{n}^{2}(\nu) \leq(1+2 c)\left(\lambda+\varepsilon_{p}\right)^{2 n}$, and hence $\varlimsup_{n \rightarrow \infty} \lambda_{n}(\nu) \leq \lambda$.

By Lemma 3 it remains to show that

$$
\varlimsup_{n \rightarrow \infty}\left|P_{n}(x, \nu)\right|^{1 / n} \leq \lambda \quad \text { for } \quad x \in \bigcup_{P=1}^{\infty} \mathscr{K}_{p} .
$$

However, if this is false there exists some positive number $\varepsilon$, some $x \in \bigcup_{P=1}^{\infty} \mathscr{K}_{p}$, and an increasing sequence of positive integers $\left\{k_{n}\right\}_{n=1}^{\infty}$ such that $\left|P_{k_{n}}(x, \nu)\right|>$ $(\lambda+\varepsilon)^{k_{n}}(n=1,2, \ldots)$. Then, if $n$ is sufficiently large and $t_{p-1}<k_{n} \leq t_{p}$,

$$
N_{k_{n}}(\nu) \geq c\left(c_{p, k_{n}}\right)\left\|P_{k_{n}}(x, \nu)\right\|_{\mathscr{K}_{p}}^{2} \geq c\left(1-\varepsilon_{p}\right)^{k_{n}}(\lambda+\varepsilon)^{2 k_{n}}
$$

(property (d)) which when $n$ tends to infinity leads to the contradiction of the inequality above.

Finally, $\underline{\lim }_{n \rightarrow \infty} \lambda_{n}(\nu) \geq \lambda$ follows from the additional fact that for monic polynomials $P_{n}(z)$ of degree $n$ and compact sets $K,\left\|P_{n}(z)\right\|_{K} \geq(C(K))^{n}[4$, p. 62].

\section{Proofs of Lemmas}

Proof of Lemma 2. For the regular compact set $\mathscr{K}$ let

$$
\mathscr{K}_{\delta}=\{x \in \mathrm{C}: \text { there exists } y \in \mathscr{K} \text { such that }|x-y| \leq \delta\} .
$$


Let $G(z, \Omega)=\log \left(C(\mathscr{K})^{-1}\right)-U\left(z, \mu_{\mathscr{K}}\right)$ be the Green's function for $\Omega$, the unbounded component of the complement of $\mathscr{K}$, and let $G_{\delta}(\Omega)=\max (G(z, \Omega))$ over $z \in \mathscr{K}_{\delta}$. We demonstrate first that there is a sequence $\left\{d_{n}\right\}_{n=1}^{\infty}$ with $\lim _{n \rightarrow \infty}\left(d_{n}\right)^{1 / n}=1$ such that

$$
\left\|P_{n}^{\prime}(z)\right\|_{\mathscr{H}_{1 / n}} \leq d_{n}\left\|P_{n}(z)\right\|_{\mathscr{H}}
$$

for a polynomial $P_{n}(z)$ of degree $n$.

Let $z \in \mathscr{K}_{1 / n}$ and let $C$ be the circle of radius $1 / n$ centered at 2 . From $P_{n}^{\prime}(z)=$ $(2 \pi i)^{-1} \int_{C} P_{n}(t)(t-z)^{-2} d t$ it follows that $\left\|P_{n}^{\prime}(z)\right\|_{\mathscr{T}_{1 / n}} \leq n\left\|P_{n}(z)\right\|_{\mathscr{T}_{2 / n}}$. By the maximum principle $\log \left|P_{n}(z)\right|-n G(z, \Omega) \leq \log \left\|P_{n}(z)\right\|_{\mathscr{K}}$ for $z \in \Omega$, and hence $\left\|P_{n}(z)\right\|_{\mathscr{H}_{2 / n}} \leq\left(\exp \left(G_{2 / n}(\Omega)\right)\right)^{n}\left\|P_{n}(z)\right\|_{\mathscr{H}}$ from which we obtain the desired inequality with $d_{n}=n\left(\exp \left(G_{2 / n}(\Omega)\right)\right)^{n}$. The regularity of $\mathscr{K}$ implies $\lim _{n \rightarrow \infty}\left(d_{n}\right)^{1 / n}=1$.

Next, assume $\left|P_{n}\left(z_{0}\right)\right|=\left\|P_{n}(z)\right\|_{\mathscr{K}}$ for some $z_{0} \in \mathscr{K}$. From $P_{n}(z)=$ $P_{n}\left(z_{0}\right)+\int_{z_{0}}^{z} P_{n}^{\prime}(t) d t$ it follows that for $z$ satisfying $\left|z-z_{0}\right| \leq \delta_{n}$ with $\delta_{n}=\left(2 d_{n}\right)^{-1}$,

$$
\begin{aligned}
\left|P_{n}(z)\right| & \geq\left|P_{n}\left(z_{0}\right)\right|-\left|z-z_{0}\right|\left\|P_{n}^{\prime}(z)\right\|_{\mathscr{K}_{1 / n}} \\
& \geq\left\|P_{n}(z)\right\|_{\mathscr{K}}\left(1-\left|z-z_{0}\right| d_{n}\right) \\
& \geq(1 / 2)\left\|P_{n}(z)\right\|_{\mathscr{K}} .
\end{aligned}
$$

Let $x_{j}=-1+j\left(\delta_{n} / 2\right)$, and let $m_{n}=\left[4 / \delta_{n}\right]+1$ (integer part). Let $I_{j}=\left[x_{j-1}, x_{j}\right]$, so $S(\mu) \subset I_{1} \cup \cdots \cup I_{m_{n}}$. Let $I_{k}, k=1,2, \ldots, k_{n}, k_{n} \leq m_{n}$, denote those intervals $I_{j}$ which intersect $\mathscr{K}$, and let $x_{k} \in \mathscr{K} \cap I_{k}$. Let $A_{k}$ be a compact subset of $B \cap$ $N_{\left(\delta_{n}\right) / 2}\left(x_{k}\right)$ with $\mu\left(A_{k}\right)>0$. Hence, if $x \in \mathscr{K}$, then $x \in I_{k}$ for some $k$, so that for any $y \in A_{k},|x-y| \leq\left|x-x_{k}\right|+\left|x_{k}-y\right| \leq \delta_{n}$.

Now let $A_{n}=A_{1} \cup \cdots \cup A_{k_{n}}$, let

$$
\tau_{n}(x)=\left(n^{2} k_{n}\right)^{-1} \sum_{k=1}^{k_{n}} \chi_{A_{k}}(x)\left(\mu\left(A_{k}\right)\right)^{-1},
$$

and let $c_{n}=\left(4 n^{2} k_{n}\right)^{-1}$.

Proof of Lemma 3. Assuming (i) it follows from the lower envelope theorem $[3$, p. 85$]$ that

$$
U\left(x, \mu_{B}\right) \leq \varliminf_{n \rightarrow \infty} U\left(x, \nu_{n}(\nu)\right)=\varliminf_{n \rightarrow \infty} \log \left|P_{n}(x, \nu)\right|^{-1 / n},
$$

where strict inequality is possible only on a set $Z_{1}$ of capacity zero. Then

$$
\varlimsup_{n \rightarrow \infty}\left|P_{n}(x, \nu)\right|^{1 / n}=\lambda \quad \text { for } \quad x \in B^{*}-Z_{1},
$$

where

$$
U\left(x, \mu_{B}\right)=\log \left(\lambda^{-1}\right) \quad \text { for } \quad x \in B^{*} \subset B, C\left(B^{*}\right)=\lambda .
$$

Assuming (ii) we have

$$
\varliminf_{n \rightarrow \infty} U\left(x, \nu_{n}(\nu)\right) \geq \log \left(\lambda^{-1}\right) \quad \text { for } \quad x \in B^{*} .
$$

Let $\nu_{0}$ be any weak limit of a subsequence of $\left\{\nu_{n}(\nu)\right\}_{n=1}^{\infty}$, say $\lim _{n \rightarrow \infty} \nu_{s_{n}}(\nu)=\nu_{0}$. It then suffices to show that $\nu_{0}=\mu_{B}[5]$. 
By the lower envelope theorem $U\left(x, \nu_{0}\right) \leq \underline{\lim }_{n \rightarrow \infty} U\left(x, \nu_{s_{n}}\right)$ where strict inequality is possible only on a set $Z_{2}$ of capacity zero, and hence $U\left(x, \nu_{0}\right) \geq$ $\log \left(\lambda^{-1}\right)$ for $x \in B^{*}-Z_{2}$. Let $K_{n}(n=1,2, \ldots)$ be compact subsets of $B^{*}-Z_{2}$ such that $K_{n} \subset K_{n+1}, C\left(K_{n}\right)>0$, and $\lim _{n \rightarrow \infty} C\left(K_{n}\right)=\lambda$. Let $\mu_{n}$ denote the equilibrium measure for $K_{n}$, and let

$$
v_{n}(x)=\left(U\left(x, \mu_{n}\right)-\log \left(C\left(K_{n}\right)^{-1}\right)\right)-\left(U\left(x, \nu_{0}\right)-\log \left(\lambda^{-1}\right)\right) .
$$

Observe that $v_{n}(x) \leq 0$ for $x \in B^{*}-Z_{2}$. Also, $v_{n}(x)$ is harmonic and bounded above on $\mathbf{C}-\left(K_{n} \cup S\left(\nu_{0}\right)\right)$ since $\lim _{x \rightarrow \infty}\left(U\left(x, \mu_{n}\right)-U\left(x, \nu_{0}\right)\right)=0$. By upper semicontinuity $\varlimsup_{x \rightarrow x_{0}} v_{n}(x) \leq 0$ for $x \in C-\left(K_{n} \cup S\left(\nu_{0}\right)\right)$ and $x_{0}$ in the boundary of $\left(K_{n} \cup S\left(\nu_{0}\right)\right)-Z_{2}$, and hence, by the generalized maximum principle [4, p. 77],

$$
v_{n}(x) \leq 0 \quad \text { for } \quad x \in \mathrm{C}-\left(K_{n} \cup S\left(\nu_{0}\right)\right) .
$$

Let $\mu_{0}$ be any weak limit of a subsequence of $\left\{\mu_{n}\right\}_{n=1}^{\infty}$, say $\lim _{n \rightarrow \infty} \mu_{t_{n}}=\mu_{0}$. Then

$$
U\left(x, \mu_{0}\right) \leq \varliminf_{n \rightarrow \infty} U\left(x, \mu_{t_{n}}\right) \leq \underline{\lim _{n \rightarrow \infty}} \log \left(\left(C\left(K_{t_{n}}\right)\right)^{-1}\right)=\log \left(\lambda^{-1}\right)
$$

for all $x$ with equality holding on $\bigcup_{n=1}^{\infty} K_{n}-Z_{3}, C\left(Z_{3}\right)=0$. It then follows from the uniqueness of an equilibrium measure that $\mu_{0}=\mu_{B}$, and hence $\lim _{n \rightarrow \infty} \mu_{n}=\mu_{B}$, and

$$
\lim _{n \rightarrow \infty} U\left(x, \mu_{n}\right)=U\left(x, \mu_{B}\right) \quad \text { for } \quad x \in \mathbf{C}-I
$$

Thus it follows that for $x \in \mathrm{C}-I$ the bounded harmonic function $U\left(x, \mu_{B}\right)-$ $U\left(x, \nu_{0}\right)$ is nonpositive, so by the maximum principle $U\left(x, \mu_{B}\right)=U\left(x, \nu_{0}\right)$ for $x \in \mathrm{C}-I$, and hence $\mu_{B}=\nu_{0}[4$, pp. 34,50$]$.

\section{Some Related Results and Some Open Questions}

If we let $\lambda_{n}(\mu)=\left(N_{n}(\mu)\right)^{1 / n}$, then we have the following results:

(a) ([5], [9]). $\underline{C}(\mu) \leq \underline{\lim }_{n \rightarrow \infty} \lambda_{n}(\mu) \leq \varlimsup_{\lim } \lambda_{n \rightarrow \infty} \lambda_{n}(\mu) \leq \bar{C}(\mu)$.

(b) ([7]). $\left\{\lambda_{n}(\mu)\right\}_{n=0}^{\infty}$ need not converge if $\underline{C}<\bar{C}$.

(c) ([7]). The limit point set of $\left\{\lambda_{n}(\mu)\right\}_{n=0}^{\infty}$ is a closed interval.

(d) ([8]). For any $[\alpha, \beta] \subset[C, \bar{C}]$ there exists a carrier related measure $\nu$ such that the limit point set of $\left\{\lambda_{n}(\nu)\right\}_{n=0}^{\infty}$ equals $[\alpha, \beta]$.

Thus if $\underline{C}=\bar{C}, \lim _{n \rightarrow \infty} \lambda_{n}=\bar{C}$, and if $\underline{C}<\bar{C}$ no further information can be obtained about the norm behavior from measurements on the carriers.

Thus the relationship between the carriers of a weight measure and the norm behavior is completely settled, and we look at the weak limits of zeros of orthogonal polynomials. In the light of the result of the present paper we have the following open questions:

(a) If $\underline{C}>0$, is every weak limit the equilibrium measure of a carrier? 
(b) If $\left\{\lambda_{n}(\nu)\right\}_{n=0}^{\infty}$ converges to $\lambda, \underline{C}<\lambda<\bar{C}$, does $\left\{\nu_{n}(\nu)\right\}_{n=1}^{\infty}$ converge?

(c) How can the weak limits that arise from one measure $\mu$ be characterized?

\section{References}

1. A. Ancona (1983): Demonstration d'une conjecture sur la capacité et effilement. C. R. Acad. Sci. Paris, Serie I, 297:393-395.

2. A. Ancona (1984): Sur une conjecture concernant la capacité et l' effilement. In: Theorie du Potential, Proceedings, Orsay 1983 (A. Dold, B. Eckmann, eds.). Lecture Notes in Mathematics, vol. 1096. New York: Springer-Verlag, pp. 10-21.

3. Ch. J. de La Vallée Poussin (1949): Le Potential Logarithmique. Paris: Gauthier-Villars.

4. M. Tsuji (1959): Potential Theory in Modern Function Theory. Tokyo: Maruzen.

5. J. L. Ullman (1972): On the regular behavior of orthogonal polynomials. Proc. London Math, Soc., 24:119-148.

6. J. L. Ullman (1985): Orthogonal polynomials for general measure, II. In: Polynômes Orthogonaux et Applications, Proceedings, Bar-le-Duc 1984 (A. Dold, B. Eckmann, eds.). Lecture Notes in Mathematics, vol. 1171. New York: Springer-Verlag, pp. 247-254.

7. J. L. Ullman, M. F. Wyneken, L. Ziegler (1986): Norm oscillatory weight measures. J. Approx. Theory, 46:204-212.

8. M. F. Wyneken (submitted): Norm asymptotics of orthogonal polynomials for general measures. Constr. Approx.

9. L. Ziegler (1977): Norm and zero asymptotics for extremal polynomials. Thesis. Ann Arbor: University of Michigan.

\section{J. L. Ullman}

Department of Mathematics University of Michigan

Ann Arbor

Michigan 48109-1003

U.S.A.
M. F. Wyneken

Department of Mathematics

University of Michigan-Flint

Flint

Michigan 48502-2186

U.S.A. 